\title{
A Study on Prevalence of Acne Vulgaris and Its Impact on Quality of Life in Adolescents of Kendriya Vidyalaya's of Jalahalli Area of Bangalore
}

\author{
Shabnam \\ Assistant Professor Markaz Unani Medical College Kozhikode Kerala
}

\begin{abstract}
Background and Objective: Acne vulgaris is a chronic condition affecting more than $85 \%$ of adolescents and young adults. It is one of the most common diseases affecting humanity and its impact on quality of life (QoL) is important. The interaction of acne and psychosocial issues is complex and, in adolescence, can be associated with developmental issues of body image, socialization and sexuality. Thus, the present study was conducted to assess the prevalence of acne vulgaris, its impact on quality of life and to study socio-demographic factors, family history and lifestyle among the selected schools in Jalahalli area of Bengaluru.

Methods: The present study was descriptive cross sectional school based study conducted in the Jallahalli area of Bengaluru city from January 2017 to December 2017. A total of 200 subjects were included. A predesigned, pretested, structured questionnaire consisting of questions on various aspects regarding socio-demographic profile, assessment of quality of life and symptoms of acne vulgaris were administered to the participants fulfilling inclusion criteria. Data regarding the severity of acne vulgaris and Quality of life status were obtained by using Global Acne Grading System and CADI and CDLQI/DLQI respectively. SPSS 18.0 software was used for data analysis.

Results: Findings of the study revealed that, Acne prevalence was $87 \%$ affecting both sexes. Self reported mild acne was present in $85.1 \%$ and moderate severe acne in $14.9 \%$ of the adolescents. The mean age of the subjects was 14.78 years. $52.5 \%$ were females and $47.5 \%$ were males. A strong relation was found between the severity of acne and quality of life $(\mathrm{p}<0.001)$.Subject with moderate/severe acne experience greater impairment in quality of life $(\mathrm{p}<0.001)$. Heredity is correlated with acne and its severity was significant.

Interpretation and Conclusion: Acne affects the quality of life of an adolescent. The impact is proportional to the severity of acne. More severe acne is associated with greater effect on quality of life. The present study reveals that increasing prevalence of Acne vulgaris and poor quality of life among the adolescents. Evaluation of quality of life in such patients may greatly help in the better management of acne, hence improving their quality of life.
\end{abstract}

Keywords: Acne vulgaris; Adolescent; Quality of life; GAGS; CDLQI/DLQI.

\section{INTRODUCTION}

Physical appearance is important in our society and influences the way in which we are perceived by others. The skin is the most visible organ of the body and determines, to a large extent, our appearance with a wide function in social and sexual communication. ${ }^{1}$ Acne vulgaris is one of the most common skin disorder. ${ }^{2,3}$ Acne vulgaris is a common skin disease affecting up to $80 \%$ of adolescents and many adults at some stage. it is associated with considerable psychological impairment which is comparable with certain chronic diseases like asthma, epilepsy, diabetes and arthritis. ${ }^{2,3}$ Acne patients are prone to low self esteem, low confidence and social dysfunction which may lead to anxiety, 
depression, obsessive compulsive disorder and sometimes suicidal ideation. ${ }^{4}$ Also prevalence of acne in school children reported ranges from $30-100 \%$ depending on age. ${ }^{5}$ More severe acne has been suggest to be associated with increased anxiety, depression symptoms and impact on patient life $^{6}$

The psychosocial affect of acne was first recognized in 1948, when Sulz Barger Zalden mentioned that there is no single disease which causes more psychic trauma and more maladjustment between parents and children more general insecurity and feeling of inferiority and greater sums of psychic assessment than does acne vulgaris. Acne is strongly associated with depression and anxiety. ${ }^{7}$

Quality of life is a general term which includes a feeling of joy and satisfaction with life. WHO defines QOL as the "individuals' perception of their position in the context of culture and value systems in which they live and in relation to their goals, expectations, standards, and concerns." 4

Acne vulgaris is a chronic inflammatory disease of the pilosebaceous follicles, common in adolescents, characterized by comedones, papules, pustules, cysts, nodules, and occasionally scars. ${ }^{9}$ The term acne is derived from Greek word 'acme' which means 'prime of life'. Although generally considered to be a benign, self limiting condition, acne may cause severe psychological problems or disfiguring scars that can persist for a lifetime. ${ }^{10}$

According to Ibn-Sina, Buthure-iLabaniyya (acne) are small white eruption on the nose and cheeks which resemble condense drop of milk. ${ }^{11}$ According to Qarshi it is a muttaaddi disease in which small white eruption appear on face nose and cheeks, on pressing a cheesy material expressed out from it. ${ }^{12}$ Causes of these eruption is a maddae-i-sadidiya which comes towards skin surface due to bukharat-i-badan. ${ }^{11}$
According to Hakeem Ajmal Khan, sometimes small pointed eruption appears on face, neck, chest, cheeks and nose. These eruptions are hard and red in color. When these eruptions become mature they excrete keel and some amount of pus. ${ }^{13}$

According to classical Unani literature, Buthur is a type of waram. The difference is only in size. Buthur are small awram. ${ }^{14}$ If any organ is unable to excrete out fudlat from skin or other organ disposed there fudlat towards this organ and organ is unable to dispose the waste, it results innutu or elevation in organ. If this elevation does not rupture the skin or mucous membrane then it is known as waram, and if the elevation is crushed it is known as buthur. Buthur which are on face and nose and are non itching, they are known as labaniyya or samdiyya. 15

\section{METHODOLOGY}

The present study entitled "A study on prevalence of Acne Vulgaris and its impact on Quality of life in adolescents of Kendriya Vidyalaya's of Jalahalli area of Bangalore "was conducted by the department of Tahaffuzi wa Samaji Tib, National Institute of Unani Medicine, Bengaluru. The aims and objectives of the study were to assess the prevalence of acne vulgaris, its impact on the quality of life and to study socio-demographic factors, family history and lifestyle associated with the disease.

\section{Criteria for selection of Subjects}

(a) Inclusion criteria:

- Subjects of 12-18 year of age

- Subjects of both genders

(b) Exclusion criteria:

- Subjects of $<12$ years and $>18$ years of age

- Subject with known dermatological condition

- Those who are not willing to participate in the study

\section{Study design}

Present study was descriptive crosssectional study to assess the prevalence of Acne Vulgaris and its impact on Quality of 
life in adolescents of Kendriya Vidyalaya's of Jalahalli area of Bangalore.

\section{Study Population}

Study population comprised of school children of age group 12-18 years (adolescents) of selected schools of Jallahalli. Children of both sexes were included.

\section{Duration of study}

The study was conducted for a period of one year, from January 2017 to December 2017.

\section{Sample size}

Sample size was calculated as 200 using formula $=\mathrm{Z}_{\alpha / 2}^{2} \times \mathrm{P} \times(1-\mathrm{P}) \times \mathrm{D} \div \mathrm{E}^{2}$

Where $\mathrm{P}$ is the prevalence or proportion of event of interest for study $(\mathrm{P}=70 \%$ taken from the previous studies), $\mathrm{E}$ is the precision or margin of error $(\mathrm{E}=10 \%$ of $\mathrm{P}), \mathrm{Z}_{\alpha / 2}$ is normal deviation at $5 \%$ level of confidence $(=1.96)$ and $\mathrm{D}$ is the design effect (which is 1 for simple random sampling)

\section{Ethical clearance}

Before starting the study, a comprehensive protocol was prepared and put for obtaining clearance from the Institutional Ethics committee (IEC) of NIUM, Bengaluru. After getting ethical clearance with IEC No: NIUM/IEC/201516/019/TST/04 study was started.

\section{Methods of collection of data}

Data was collected from selected schools of Jallahalli using simple random sampling method. Respondents were enrolled in the study after taking written assent/consent from them. A pretested structured questionnaire consisting various questions and aspects regarding sociodemographic profile, acne vulgaris and the assessment of quality of life were administered to the respondents fulfilling inclusion criteria. Quality of life assessment data were obtained by using CADI, CDLQI/DLQI and Acne grading was calculated by using GAGS.

Socio-economic status: Socioeconomic status of the parents was calculated by using Kuppuswamy's socioeconomic status scale 2014. On the basis of that parents were graded in different socioeconomic classes as upper class (I), upper middle class (II), lower middle class (III), upper lower class (IV) and lower class (V).

\section{Measurement of the study variables}

Age: the age of children was recorded from school register.

Sex: Gender of the included children was obtained from school record.

Language spoken: This information was gained to know whether the students require any interpreter or translated for the talk.

Assessment of Acne Vulgaris in school children; Acne was graded in to mild, moderate and sever based on GAGS (Global acne grading system) this system divides the face, chest and upper back into six areas (forehead, each cheeks, nose, chin and chest and upper back) and assign a factor to each area on the basis of size.

\begin{tabular}{lc}
\hline \multicolumn{2}{c}{ Table 3: The global acne grading system ${ }^{[12]}$} \\
\hline Location & Factor \\
\hline Forehead & 2 \\
Right cheek & 2 \\
Left cheek & 2 \\
Nose & 1 \\
Chin & 1 \\
Chest and upper back & 3 \\
\hline Note: Each type of lesion is given a value depending on severity: no \\
lesions $=0$, comedones $=1$, papules $=2$, pustules $=3$ and nodules $=4$. \\
The score for each area (Local score) is calculated using the formula: Local \\
score $=$ Factor $x$ Grade $(0-4)$. The global score is the sum of local scores, and \\
acne severity was graded using the global score. A score of $1-18$ is considered \\
mild; $19-30$, moderate; $31-38$, severe; and $>39$, very severe
\end{tabular}

CDLQI is a general questionnaire to assess the quality of life. It consist of 10 questions about disease symptoms and feelings (question 1\&2), Leisure (question $4,5 \& 6$ ), school or holidays (question 7), Personal relationships (question $3 \& 8$ ), sleep (question 9) and treatment (question10). The scoring of each question is as; Very much $=3$, Quite a lot $=2$, Only a little $=1$, Not at all $=0$, Question unanswered $=0$, Question 7: "Prevented school" $=3$.Its domain is from zero(without any effect on quality of life) to 30(extremely large effect on quality of life).The CDLQI was calculated by summing the score of 
each question resulting in a maximum of 30 and a minimum of 0 . The higher the score, the more quality of life is impaired.

Interpretation; $0-1=$ No effect on child's life, 2-6 $=$ Small effect, 7-12 = Moderate effect $13-18=$ Very large effect, $19-30=$ extremely large effect.

DLQI consists of 10 questions about disease symptoms and feeling (question $1 \& 2$ ), daily activities (question 3\&4), Leisure (question 5\&6), work and school (question 7), Personal relationships (question 8\&9), treatment (question 10).The scoring of each question is as; Very much $=$ 3 , Quite a lot $=2$, Only a little $=1$, Not at all $=0$, Question unanswered $=0$, Question 7: Question 7: "Prevented work or studying" $=3$.Its domain is from zero(without any effect on quality of life) to 30(extremely large effect on quality of life).The CDLQI was calculated by summing the score of each question resulting in a maximum of 30 and a minimum of 0 . The higher the score, the more quality of life is impaired.

Interpretation; $0-1=$ No effect on patient's life, $2-5=$ Small effect, 6-10 = Moderate effect $12-20=$ Very large effect, $21-30=$ extremely large effect.

CADI a questionnaire which was specific for acne and contains 5 questions. Each question is scored from 0-3 leading to a total score of $0-15$. The CADI score is calculated by summing the score of each question resulting in a possible maximum of 15 and minimum of 0 . CADI score is graded as low (0-4), medium (5-9) and high (1015).A higher score shows a very large impact on quality of life

Both CADI and CDLQI/DLQI questionnaire were copyright protected, hence prior permission was granted from the University and authors before their use.

CDLQI was administered to adolescents age less than 16 year of age, where as DLQI was administer to adolescent age 16 or above.

\section{RESULT}

A total number of 200 patients aged 11 to 18 years were taken in study among. Patients were divided into 4 groups in which maximum $56.5 \%$ were among patients between $15-16$ years followed $32.5 \%$ of age group 13 to 14 years and $7 \%$ patients of age group 17-18 years. Out of total number of 200 patients, $46 \%$ of patients were male and $54 \%$ of patients were female. In this study, out of 200 adolescent $36 \%$ adolescent were dark and $64 \%$ were fair complexion. $69 \%$ were non vegetarian and $31 \%$ were vegetarian. (Table1).

\begin{tabular}{|c|c|c|c|c|}
\hline \multirow[t]{2}{*}{ Demographic variables } & \multicolumn{2}{|c|}{ Acne vulgaris } & \multirow{2}{*}{$\begin{array}{l}\text { Total } \\
(\mathbf{n}=\mathbf{2 0 0})\end{array}$} & \multirow[t]{2}{*}{$P$ value } \\
\hline & YES & NO & & \\
\hline \multicolumn{5}{|l|}{ Age in years } \\
\hline $11-12$ & $6(3.5 \%)$ & $2(7.7 \%)$ & $8(4 \%)$ & \multirow[t]{4}{*}{0.202} \\
\hline $13-14$ & $57(32.7 \%)$ & $85(30.8 \%)$ & $65(32.5 \%)$ & \\
\hline $15-16$ & $101(58.1 \%)$ & $12(46.1 \%)$ & $113(56.5 \%)$ & \\
\hline $17-18$ & $10(5.7 \%)$ & $4(15.4 \%)$ & $14(7 \%)$ & \\
\hline \multicolumn{5}{|l|}{ Complexion } \\
\hline Dark & $55(31.6 \%)$ & $17(65.4 \%)$ & $72(36 \%)$ & \multirow[t]{2}{*}{0.001} \\
\hline Fair & $119(68.4 \%)$ & $9(34.6 \%)$ & $128(64 \%)$ & \\
\hline \multicolumn{5}{|l|}{ Type of Family } \\
\hline Joint & $13(7.5 \%)$ & $6(23.1 \%)$ & $19(9.5 \%)$ & \multirow[t]{2}{*}{0.029} \\
\hline Nuclear & $161(92.5 \%)$ & $20(76.9 \%)$ & $181(90.5 \%)$ & \\
\hline \multicolumn{5}{|l|}{ Socio Economic Status } \\
\hline Upper Class & $154(88.5 \%)$ & $19(73.1 \%)$ & $173(86.5 \%)$ & \multirow[t]{4}{*}{0.001} \\
\hline Upper Middle & $17(9.78 \%)$ & $5(19.2 \%)$ & $22(11 \%)$ & \\
\hline Lower Middle & $2(1.15 \%)$ & $2(7.7 \%)$ & $4(2 \%)$ & \\
\hline Upper Lower & $1(0.5 \%)$ & $0(0.0 \%)$ & $1(0.5 \%)$ & \\
\hline \multicolumn{5}{|l|}{ Food Habits } \\
\hline Non Veg & $120(68.9 \%)$ & $18(69.2 \%)$ & $138(69 \%)$ & \multirow[t]{2}{*}{1.000} \\
\hline Veg & $54(31.1 \%)$ & $8(30.8 \%)$ & $62(31 \%)$ & \\
\hline \multicolumn{5}{|l|}{ Gender } \\
\hline Female & $94(54.0 \%)$ & $11(42.3 \%)$ & $105(52.5 \%)$ & \multirow[t]{2}{*}{0.2973} \\
\hline Male & $80(46.0 \%)$ & $15(57.7 \%)$ & $95(47.5 \%)$ & \\
\hline
\end{tabular}


Shabnam. A study on prevalence of acne vulgaris and its impact on quality of life in adolescents of Kendriya Vidyalaya's of Jalahalli area of Bangalore.

Table 2: CADI distribution according to gender of Respondents

\begin{tabular}{|l|l|l|l|}
\hline \multirow{2}{*}{ CADI } & Gender & \multirow{2}{*}{ Total } \\
\cline { 2 - 3 } & Female & Male & \\
\hline Low & $33(35.1 \%)$ & $38(47.5 \%)$ & $71(40.8 \%)$ \\
\hline Medium & $42(44.7 \%)$ & $35(43.75 \%)$ & $77(44.3 \%)$ \\
\hline High & $19(20.2 \%)$ & $7(8.75 \%)$ & $26(14.9 \%)$ \\
\hline Total & $94(100 \%)$ & $80(100 \%)$ & $174(100 \%)$ \\
\hline
\end{tabular}

According to CADI scores of acne showed low effect in $40.8 \%$ of the patients, majority had medium effect in $44.3 \%$ of patients and high effect was seen on $14.9 \%$ of patients (Table2).

According to DLQI scores of acne showed no effect in $9.8 \%$ of the patients, small effect in $13.2 \%$ of the patients, moderate effect in $22.9 \%$ of patients, very large effect in $20.7 \%$ of patients and extremely large effects on $32.8 \%$ of patients. (Table 3).

Table 3: CDLQI distribution according to of Respondents

\begin{tabular}{|l|l|l|l|}
\hline \multirow{2}{*}{ CDLQI } & Gender & \multirow{2}{*}{ Total } \\
\cline { 2 - 3 } & Female & Male & $17(9.8 \%)$ \\
\hline No Effect & $7(7.4 \%)$ & $10(12.5 \%)$ & $17 \%)$ \\
\hline Low effect & $1(1.1 \%)$ & $0(0 \%)$ & $1(0.6 \%)$ \\
\hline Small Effect & $11(11.7 \%)$ & $12(15.0 \%)$ & $23(13.2 \%)$ \\
\hline Moderate effect & $20(21.3 \%)$ & $20(25.0 \%)$ & $40(22.9 \%)$ \\
\hline Very Large Effect & $19(20.2 \%)$ & $17(21.3 \%)$ & $36(20.7 \%)$ \\
\hline Extremely large effect & $36(38.3 \%)$ & $21(26.2 \%)$ & $57(32.8 \%)$ \\
\hline Total & $94(100 \%)$ & $80(100 \%)$ & $174(100 \%)$ \\
\hline \multicolumn{2}{|l|}{} \\
\hline
\end{tabular}

Table 4: CDLQI/DLQI distribution according to GAGS of respondents

\begin{tabular}{|l|l|l|l|l|l|}
\hline \multirow{2}{*}{ CDLQI/DLQI } & GAGS & \multirow{2}{*}{ Total } \\
\cline { 2 - 5 } & Mild & Moderate & Severe & Very Severe & \\
\hline No Effect & $17(11.7 \%)$ & $0(0 \%)$ & $0(0 \%)$ & $0(0 \%)$ & $17(9.8 \%)$ \\
\hline Low effect & $1(0.6 \%)$ & $0(0 \%)$ & $0(0 \%)$ & $0(0 \%)$ & $1(0.6 \%)$ \\
\hline Small Effect & $23(15.5 \%)$ & $0(0 \%)$ & $0(0 \%)$ & $0(0 \%)$ & $23(13.2 \%)$ \\
\hline Moderate effect & $40(27.0 \%)$ & $1(5.3 \%)$ & $0(0 \%)$ & $0(0 \%)$ & $41(23.6 \%)$ \\
\hline Very Large Effect & $32(21.6 \%)$ & $2(10.5 \%)$ & $0(0 \%)$ & $0(0 \%)$ & $34(19.5 \%)$ \\
\hline Extremely large effect & $35(23.6 \%)$ & $16(84.2 \%)$ & $3(100 \%)$ & $4(100 \%)$ & $58(33.3 \%)$ \\
\hline Total & $148(100 \%)$ & $19(100 \%)$ & $3(100 \%)$ & $4(100 \%)$ & $174(100 \%)$ \\
\hline
\end{tabular}

This table shows that there is close association in between the severity of acne and quality of life.

\section{DISCUSSION}

The overall prevalence of acne in this study was $87 \%$ and it was more common in females than males, confirming our results with previous findings that acne is common in adolescent females. ${ }^{16}$ However the prevalence of acne was reported to be lower in some studies. ${ }^{17}$ The difference in prevalence rates between these two groups of studies may reflect ethnic variation. Comparison of prevalence rates between studies is also hampered by the varied method of acne grading used by different studies and the wide range of diagnostic criteria used.

In present study maximum number of subjects $56.5 \%$ (113) were in the age group of 15-16 years, Our finding is in accordance with the finding of a study conducted by Jancovic et al which showed that acne prevalence is more at the age of 16 and 17 years. $^{18}$ Balakrishnan et al also reported that acne is a chronic disease affecting $85 \%$ of the teenagers. ${ }^{19}$
Our study subjects comprised predominantly of females $52.5 \%$ (105) while remaining $47.5 \%(95)$ were males in the present study (table and figure no.2).Our finding are in accordance with the finding of previous studies conducted by Ismail et al in which the majority of the study population was constituted by females. ${ }^{20}$ In one study conducted by Chinese in 2012 which reported that acne in adolescents was predominant in males and adult acne was common in females. ${ }^{21}$

In our study the vast majority of students had mild acne $85.1 \%$ (148) and $10.9 \%$ (19) had moderate acne, $1.7 \%$ (3) had severe and $2.3 \%$ (4) had very severe acne (table 2). A similar pattern was obtained in comparative studies previously conducted by Yahya et al. Study conducted by Kaduna et al and Hanisha et al ${ }^{16}$ in Malaysian adolescents, recorded $93.1 \%$ and $90.2 \%$ frequency of mild acne respectively. However, Tan et al ${ }^{22}$ obtained a near equal prevalence of moderate/severe acne $(48.6 \%)$ and mild acne (51.4\%) in Singaporean adolescents. In one hospital-based studies, Agheai et al found more moderate/severe grades of acne (84\%) compared to mild 
grades (16\%), and so did Mallon et al ${ }^{23}$. In the UK this is not unexpected, as persons with more severe diseases are likely to seek medical intervention, thus hospital-based studies are likely to yield more severe forms of acne compared to community-based ones such as ours.

The overall CADI score was $44.3 \%$ (77), indicating a mild degree of disability from acne. Similar values were obtained in some community-based studies ${ }^{16}$, while higher scores were obtained by Motley and Finlay ${ }^{24}$ in the UK and Oakley ${ }^{22}$ in New Zealand, respectively. Several reasons may be adduced for the relatively higher CADI scores in the two latter studies, which were hospital-based: Firstly, hospital-based studies have a higher concentration of persons with more severe grades of acne compared to community-based surveys. Secondly, the ages of patients in hospitalbased surveys are varied compared to the exclusively adolescent age of fore mentioned community-based studies. Another factor may be cultural and/or racial; the studies with higher CADI scores were conducted in Western societies. ${ }^{24}$

Our study showed that the impact of acne on quality of life was proportional to acne severity. These results are in agreement with similar studies where both acne and severity are self reported. ${ }^{25}$, $23,26,27,28,16,29,30$. It was observed that there was significant correlation between the CADI and GAGS $(p<0.001)$.Our finding is in accordance with the finding of previous studies conducted by Hanisha et al ${ }^{16}$ who recorded that the severity of acne correlate strongly with the effect of quality of life. But in some other studies the correlation between GAGS and CADI was weak as reported by Law et $a l^{35}$.

In the present study it was observed that there was a moderate to extremely large effect based on CDLQI/DLQI score which is similar to study by Safizedeh $\mathrm{H}$ et al. who found that there was a moderate to very large influence on quality of life in patients with acne. Haritha et al also found moderate to very large effect of acne in adolescents (table 3).

In present study it was observed that there was medium effect $44.3 \%$ (77) of Acne vulgaris on adolescents based on CADI score which indicate high psychosocial burden from acne. Similarly, Hanish et al reported that based on specific response of CADI of the patients, they felt aggressive, frustrated or embarrassed as a result of having acne. ${ }^{16}$

A study done in the psychiatry OPD of a medical institution in New Delhi found significantly higher psychiatric morbidity in patient with acne vulgaris. Similarly, in our study a significant correlation of GAGS score with CDLQI/DLQI $(\mathrm{p}<0.001)$ and CADI $(p<0.001)$ was found. The result showed that acne has an impact on quality of life. ${ }^{36}$

\section{Study's Limitations}

This study has a few limitations. First, the study was limited to one geographical location (Jalahalli) hence; the result of the study cannot be generalized to the entire city. Second issue is of over reporting, which is a well-recognized issue for self-report surveys as the participants tend to report in socially desirable ways. For example, the smoker may want to overreport non smoker to appear healthier. Third, it is a cross sectional study design which limits causality of relations. Further sample size was less compared with many of the other studies.

\section{CONCLUSION}

Thus, it may be concluded that evaluation of the quality of life in patients with acne is important as it help in the pharmacological as well as psychological treatment of these patients in a more effective and integrated way. Further Health education is needed in our secondary schools to ensure that adolescents understand their disease and to know what treatments are available and from whom they should seek advice. A Health professional should be aware that early acne 
treatment can prevent progression of the disease and its complication.

\section{Acknowledgement: None}

\section{Conflict of Interest: None}

\section{Source of Funding: None}

\section{Ethical Approval: Approved}

\section{REFERENCES}

1. Vilar GN, Santos LA, Filho JFS. Quality of life, self-esteem and psychosocial factors in adolescents with acne vulgaris. A Bras Dermatol. 2015; 90 (5):622-9.

2. Behn PN,Aggarwal A,Srivastava G.Practice of dermatology.Edi 9 New Delhi: CBS Publishers and distributors 2002:408-411

3. Mcphee SJ, Papadakis MA. Lange current Medical Dignosis and Treatment. Edi 49.New Delhi: McGraw Hill Medical,2010:120-122.

4. Samanthula H, Kodali M. Acne and quality of life- A study from a tertiary care centre in south India. Journal of dental and medical science.2013; 6(3);59-62.

5. Yeung C, Teo LHY, Xiang LH, Chan HHL. A community based epidemiological study of acne vulgaris in Hong Kong adolescents. ACTA Derm Venereol. 2002; 82: 104-107.

6. Tasoula E, Gregorious S, Chalikiyas J, Lazarou D, Danopoulous I, Katsambas A et $a l$. The impact of acne vulgaris on quality of life and psychic health in young adolescent in Greece.Result of population survey. An bras Dermatol 2012; 87(6):863-867.

7. Rubin MG, Kim K, Logan AC. Acne vulgaris mental health and omega-3 fatty acids: A Report of cases. Lipid in health and disease 2008;7(36):1-3

8. Ghaderi R, Saadatjoo A, Ghaderi F. Evaluating of life quality in patients with acne vulgaris using generic and specific questionnaires. Dermatology research and practice. 2013; 1-6.

9. Bagatin E, Timpano DL, Guadanhim LRD, Nogueira VMA, Terzian LR, Steiner D et al. Acne vulgaris: prevalence and clinical forms in adolescents from São Paulo, Brazil. An Bras Dermatol. 2014; 89(3):428-35.

10. Tahir MC. Pathogenesis of acne vulgaris simplified. Journal of Pakistan association of dermatologist 2010;20-93-96
11. Ibne sina Abu Ali. Al Qanoon Fil Tib(urdu translation by kantoori sayed ghulam hasnain) New Delhi:Idara kitab Us shifa, YNM:1432.

12. Qurshi Hasan Mohammad, Jamiul Hikmat. New Delhi:Idara kitabushifa 2011:994,995.

13. Jeelani Ghulam. Makhzane Hikmat. Vol.2. New Delhi: Ejaz Publication, 1996:689

14. Almaseehi, Ibne Alqaf AFA. Kitabal Umda Fil jarahat. Vol1. New Delhi: CCRUM;YNM:146

15. Siddiqi MMH, Jafari AHS. Tarjuma Daqa-iQulilaaj.vol.1.Deoband: Mukhtar press; YNM: 478-80.

16. Hanisah A, Omar K, Shah SA, "Prevalence of acne and its impact on the quality of life in school -aged adolescents in Malaysia," Journal of Primary Health Care, 2009; 1:2025.

17. Aktan S, Ozmen E, Sanli B. Anxiety, depression, and nature of acne vulgaris in adolescents. Int $J$ Dermatol. 2000; 39 (5):354-357.

18. Jankovic S, Vukicevic J, Djordjevic S, Jankovic J, Marinkovic J. Quality of life among schoolchildren with acne: Results of a Crosssectional study. Indian J Dermatol Venereol Leprol 2012; 78:454-8.

19. Balkrishnan R, Kulkarni AS, Cayce K, et al. Predictors of healthcare outcomes and costs related to medication use in patients with acne in the United States. Cutis 2006 Apr; 77(4):251-5.

20. Ismail KH, Mohammad Ali KB. Quality of life in patients with acne in Erbil city. Health and quality of life outcomes2012; 10(60):1, 2, 4.

21. Yiwei S, Wang T, Zhou C. Prevalence of Acne Vulgaris in Chinese Adolescents and Adults: A Community-based Study of 17,345 Subjects in Six Cities: Acta Derm Venereol 2012; 92: 40-44.

22. Oakley AM. The Acne Disability Index: usefulness confirmed. Australas J Dermatol. 1996; 37(1):37-39.

23. Mallon E, Newton JN, Klassen A, StewartBrown SL, Ryan TJ, Finlay AY. The quality of life in acne: a comparison with general medical conditions using generic questionnaires. $\quad \mathrm{Br}$ J Dermatol. 1999; 140(4):672-676.

24. Motley RJ, Finlay AY. Practical use of a disability index in the routine management of acne. Clin Exp Dermatol. 1992; 17 (1):13. 
25. Walker N, Lewis Jones MS. Quality of life and acne in Scottish adolescent children: use of the Children's Dermatology Life Quality Index (CDLQI) and the Cardiff Acne Disability Index (CADI). J Eur Acad Dermatol Venereol. 2006; 20:45-50.

26. Poli F, Dreno B, Verschoore M: An epidemiological study of acne in female adults: Results of a survey conducted in France. JEADV 2001, 15:541-545.

27. Pawin H, Chivot M, Beylot C, Faure $M$, Poli F, Revuz J, Dréno B. Living with acne. A study of adolescents' personal experiences. Dermatology. 2007; 215:30814.

28. Finlay AY and Khan GK, Dermatology life quality index (DLQI) A simple practical measure for routine clinical use clinical and experimental Dermatology volume 19 no 3.1994; pp. 210-216.

29. Abdel-Hafez K, Mahran AM, Hofny ER, Mohammed KA, Darweesh AM, Aal AA. The impact of acne vulgaris on the quality of life and psychological status in patient from upper Egypt. Int J Dermatol. 2009; 48:280-5.

30. Gupta MA, Gupta AK. Psychiatric and psychological co-morbidity in patients with dermatologic disorders: epidemiology and management. Am J Clin Dermatol.2003; 4:833-42.

31. Ilgen E, Derya A. There is no correlation between acne severity and AQOLS/DLQI scores. J Dermatol. 2005; 32:705-10.
32. Girman CJ, Hartmaier S, Thiboutot D, Johnson J, Barber B, DeMuro-Mercon C, et al. Evaluating health related quality of ife in patients with facial acne: development of a self administered questionnaire for clinical trials. Qual Life Res. 1996; 5:131-8.

33. Niemeier V, Kupfer J, Demmelbauer-Ebner M, Stangier U,Effndy I, Gieler U. Coping with acne vulgaris. Evaluation of the Chronic Skin Disorder Questionnaire with acne. Dermatology. 1998; 196:108-15.

34. Salek MS, Khan GK, Finlay AY. Questionnaires techniques in assessing acne handicap: reliability and validity study. Qual Life Res. 1996; 5:131-8.

35. Law MP, Chuh AA, Lee A, Molinary N.Acne prevalence and beyond: acne disability and its predictive factors among Chinese late adolescent in hong kong.clin Exp dermatol 2010; 35(1):16-21.

36. Kokandi A, Evaluation of acne quality of life and clinical severity in acne female adults. Dermatology research practice; doi 10.1155/2010/410809 (2010).

How to cite this article: Shabnam. A study on prevalence of acne vulgaris and its impact on quality of life in adolescents of Kendriya Vidyalaya's of Jalahalli area of Bangalore. Int $J$ Health Sci Res. 2021; 11(9): 33-40. DOI: https://doi.org/10.52403/ijhsr.20210905 